\title{
Urinary Metabolites of Organophosphate Flame Retardants and Their Variability in Pregnant Women
}

\author{
Kate Hoffman ${ }^{1,2}$, Julie L. Daniels ${ }^{2}$, and Heather M. Stapleton ${ }^{1}$ \\ Kate Hoffman: kate.hoffman@duke.edu; Julie L. Daniels: julie_daniels@unc.edu; Heather M. Stapleton: \\ heather.stapleton@duke.edu \\ ${ }^{1}$ Nicholas School of the Environment, Duke University, LSRC, Box 90328, Durham, NC 27708 \\ USA \\ ${ }^{2}$ Gillings School of Global Public Health, University of North Carolina, 135 Dauer Drive, Campus \\ Box 7435, Chapel Hill, NC 27599 USA
}

\begin{abstract}
Organophosphate flame retardants (OPFRs) are commonly added to consumer products to reduce their flammability. Based on levels of OPFRs in indoor environments, human exposure is likely chronic and ubiquitous. Animal studies suggest that exposure to some OPFRs may result in adverse health impacts, particularly for tris (1,3-dichloropropyl) phosphate (TDCPP); however, human data on the impacts of exposure to OPFRs are lacking. To design human studies, more information is needed on the stability of measured OPFRs in human samples over time. In this study, we sought to assess the degree of temporal variability of urinary TDCPP and triphenyl phosphate (TPP) metabolites throughout pregnancy in a cohort of women from central North Carolina.
\end{abstract}

Eight pregnant women provided multiple urine samples: 3 during the $18^{\text {th }}$ week of pregnancy, 1 during the $28^{\text {th }}$ week, and 1 shortly after the child's birth. Bis (1,3-dichloropropyl) phosphate (BDCPP) and diphenyl phosphate (DPP), the respective metabolites of TDCPP and TPP, were measured in urine samples using liquid chromatography-tandem mass spectrometry. BDCPP and DPP were each detected in 38 of 39 urine samples and were not normally distributed. Geometric mean BDCPP and DPP concentrations were $1.3 \mathrm{ng} / \mathrm{mL}$ (interquartile range (IQR): $0.8,2.7 \mathrm{ng} / \mathrm{mL}$ ) and $1.9 \mathrm{ng} / \mathrm{mL}$ (IQR: $0.9,3.5 \mathrm{ng} / \mathrm{mL}$ ), respectively. BDCPP and DPP were moderately to strongly reliable over one week (ICC $=0.5 ; 95 \%$ confidence interval $(\mathrm{CI}): 0.4,0.7$ and $\mathrm{ICC}=0.7 ; 95 \% \mathrm{CI}$ : $0.5,0.8$, respectively), and over the entire pregnancy (ICC $=0.595 \% \mathrm{CI}: 0.3,0.7$ and $\mathrm{ICC}=0.6$; 95\% CI: 0.4, 0.7, respectively). These data suggest that exposures to TDCPP and TPP are widespread and variable for pregnant women, and that a single measure of BDCPP or DPP, taken in the second trimester, likely captures information on the rank order of exposure throughout pregnancy.

\footnotetext{
(C) 2013 Elsevier Ltd. All rights reserved.

Corresponding Author: Kate Hoffman, Nicholas School of the Environment, Duke University, LSRC Box 90328, Durham, NC 27708 USA, phone: (919) 684-6952, fax: (919) 684-8741, kate.hoffman@ duke.edu.

Publisher's Disclaimer: This is a PDF file of an unedited manuscript that has been accepted for publication. As a service to our customers we are providing this early version of the manuscript. The manuscript will undergo copyediting, typesetting, and review of the resulting proof before it is published in its final citable form. Please note that during the production process errors may be discovered which could affect the content, and all legal disclaimers that apply to the journal pertain.
} 


\section{Keywords}

organophosphate flame retardants (OPFRs); urine; variability; pregnant women; tris (1, 3dichloropropyl) phosphate (TDCPP); triphenyl phosphate (TPP)

\section{Introduction}

Flame retardant chemicals have been added to a variety of household products to meet flammability standards for many years. Until recently, polybrominated diphenyl ethers (PBDEs) accounted for a large proportion of flame retardants used in household products, including polyurethane foam and electronics; however, regulatory action and concern over the persistence, bioaccumulation, and toxicity of PBDEs led to their phase-out and the introduction of alternative flame retardants (Stapleton, Allen et al. 2008, van der Veen and de Boer 2012). Organophosphate flame retardants (OPFRs) such as tris (1,3-dichloropropyl) phosphate (TDCPP) and triphenyl phosphate (TPP) are now among the most commonly used PBDE alternatives in residential furniture (US Environmental Protection Agency (EPA) 2005, Stapleton, Klosterhaus et al. 2009, Stapleton, Sharma et al. 2012, van der Veen and de Boer 2012). In fact, two recent studies demonstrated that TDCPP was the most commonly applied flame retardant in residential furniture and baby products containing polyurethane foam (Stapleton, Klosterhaus et al. 2011, Stapleton, Sharma et al. 2012). In addition, TDCPP and TPP have been detected with high frequency in dust collected from homes, offices, and automobiles suggesting that the majority of the population receives chronic exposure to these compounds (Meeker and Stapleton 2010, Carignan, McClean et al. 2013). Inadvertent ingestion of house dust has been shown to be a primary route of human exposure to PBDE flame retardants, and may be similar for OPFRs. Two recent assessments have reported ubiquitous detection of urinary OPFR metabolites in the general population (Carignan, McClean et al. 2013, Meeker, Cooper et al. 2013).

Toxicological data suggests that certain OPFRs may be reproductive toxins and may also have carcinogenic and neurotoxic properties (National Research Council (NRC) 2000, Dishaw, Powers et al. 2011, van der Veen and de Boer 2012). An exposure assessment conducted by the Consumer Product Safety Commission referred to TDCPP as a suspected carcinogen, and established an acceptable daily dose of 5 micrograms $/ \mathrm{kg}$ body weight per day (Babich 2006). Currently, available data are very limited on human exposure or potential human health effects. Epidemiologic studies, while critically need, are complicated by the potentially episodic nature of exposure and the short biological half-lives of OPFRs. Variations in behaviors and movement between different micro-environments may create within-person variability of OPFRs making it difficult to accurately assess human exposures. Investigations of prenatal exposures are further complicated by pregnancyrelated changes in metabolism that may contribute to variability in metabolite levels throughout gestation (Mahalingaiah, Meeker et al. 2008, Braun, Kalkbrenner et al. 2011, Braun, Smith et al. 2012).

In our present work, we utilize recently developed methods to extract and measure bis (1,3dichloropropyl) phosphate (BDCPP) and diphenyl phosphate (DPP), the respective metabolites of TDCPP and TPP, in urine samples from a group of pregnant women (Cooper, Covaci et al. 2011). We investigate both the short- and long-term variability in the levels of OPFR metabolites in urine throughout pregnancy. This information will help inform exposure assessment in epidemiological studies and aid in the interpretation of results using single exposure measures to capture information on exposure throughout pregnancy. 


\subsection{Materials and methods}

\subsubsection{Study Population}

We recruited 8 pregnant women between December 2011 to May 2012 using flyers placed in health centers, doctor's offices, and in public settings around Chapel Hill, North Carolina. Women provided demographic information and urine samples during pregnancy. In the $18^{\text {th }}$ week of pregnancy each woman collected a 24-hour urine specimen as well as 2 first morning voids, approximately 48 -hours apart. Women were also asked to provide a first morning void in the $28^{\text {th }}$ week of pregnancy and a spot urine sample after their child's birth. All 8 women provided urine samples during the first 4 sample collection time points and 7 women provided urine samples after the birth of their child (samples collected 1 to 10 days post-birth). Our final sample consisted of 39 urine specimens. All study protocols were approved by the institutional review board at the University of North Carolina-Chapel Hill and all women provided informed consent.

\subsubsection{OPFR Analysis}

Urine samples were collected in sterile polyethylene specimen containers. Samples were packed on ice and transported to the lab each day, where they were aliquoted into 15-ml conical centrifuge tubes and frozen at $-80^{\circ} \mathrm{C}$ until analysis. Extraction and measurement methods have been described previously (Cooper, Covaci et al. 2011). Briefly, BDCPP and DPP were measured in urine samples using mixed-mode anion exchange solid phase extraction and a mass-labeled internal standard (d10-BDCPP and d10-DPP) with analysis by atmospheric pressure chemical ionization liquid chromatography tandem mass spectrometry (Cooper, Covaci et al. 2011). For quality assurance purposes we evaluated the recovery of d10-BDCPP and d10-DPP in all samples, and measured the amount of BDCPP and DPP levels in laboratory blanks $(\mathrm{n}=6)$. The average recoveries of d10-BDCPP and d10-DPP were $72 \pm 13$ and $62 \pm 8 \%$, respectively. Very small amounts of DPP, were detected in laboratory blanks, while BDCPP was not detected. Therefore, the method detection limit (MDL) was calculated using three times the standard deviation of the blanks normalized to the urine volume extracted, which was $4.7 \mathrm{pg} / \mathrm{mL}$ and $12.7 \mathrm{pg} / \mathrm{mL}$, respectively. Levels of BDCPP and DPP in urine were corrected for recovery of the mass labeled internal standards. We explored several methods of imputing values below the method limit of detection, including the MDL/2 and the MDL/ $\sqrt{ } 2$ (Hornung and Reed 1990). Because results were very similar using alternate methods, we have chosen to present results using the MDL/ $\sqrt{2}$ for missing values.

Sample specific gravity (SG) was measured in each urine sample prior to analysis using a digital handheld refractometer (Atago, Bellevue, WA, USA). All analyses were conducted using both uncorrected and specific gravity adjusted concentrations account for urine dilution (Boeniger, Lowry et al. 1993). One participant had very dilute urine ( $\mathrm{SG}=1.004)$. To assess the robustness of analyses to including information from this sample we conducted sensitivity analyses omitting the observation. As excluding the observation had little impact on results, it was retained in all presented analyses.

\subsubsection{Statistical Analyses}

1.2.3.1 Descriptive Analyses-All statistical analyses were performed in SAS (SAS version 9.2; SAS institute, Cary NC). We calculated descriptive statistics for each metabolite including the geometric mean concentration and selected percentiles $\left(10^{\text {th }}, 25^{\text {th }}, 50^{\text {th }}, 75^{\text {th }}\right.$ and $90^{\text {th }}$ percentiles). We also examined the distribution of urinary BDCPP and DPP graphically. Urinary metabolite levels were skewed right. Accordingly, all quantitative analyses were conducted with natural log-transformed urinary BDCPP and DPP measures. 
We assessed the relationship between urinary BDCPP and DPP concentrations by calculating spearman correlation coefficients.

1.2.3.2 Variability Assessment-We assessed variability in BDCPP and DPP in three ways. First, we estimated the short- and long-term variability in BDCPP and DPP concentrations by calculating interclass correlation coefficients (ICCs). ICCs provide a measure of the reliability of repeated measures over time and are calculated by taking the ratio of the between-subject variability to the sum of the between- and within-subject variability (Rosner 2000). ICC values range from 0 , indicating no reproducibility, to 1 , indicating perfect reproducibility. ICCs and $95 \%$ confidence intervals were calculated using a SAS Marco developed by Hamer (1995), and based on the work of Shrout and Fleiss (1979). ICCs were calculated for samples collected in the 18th week of pregnancy to capture short-term reliability ( 3 samples each in 8 women; $n=24$ ) and for all sample points to capture long-term reliability. In analyses of long-term variability, the three samples collected from each woman during the 18th week of pregnancy were first averaged to avoid inflating ICCs with measurements taken over a short period of time, and then compared to the samples taken at $28^{\text {th }}$ week of pregnancy and at birth.

In addition to ICCs, we also conducted analyses to determine how well OPFR metabolite concentrations from each time point (18 week average, $28^{\text {th }}$ week, and birth samples) captured the rank order of exposure throughout pregnancy. First, each woman's geometric mean metabolite concentration was calculated and women were ranked (GM of 18 week average, $28^{\text {th }}$ week, and birth samples). Women with the 4 highest GM concentrations were classified as the "high" exposure group (true high exposure category). Women were then ranked at each time point based on the concentration in that individual sample (predicted exposure category). Contingency tables were constructed for each time point comparing the predicted exposure category to the true high exposure category. Contingency tables form each time point were then combined into a single table and sensitivity, specificity, positive predictive value (PPV) and negative predictive value (NPV) were calculated.

\subsection{Results}

Women participating in our study ranged in age from 28 to 36 years at conception and were predominantly white (88\%) and highly educated ( $88 \%$ had completed graduate education). All mothers delivered healthy, full-term infants. Both BDCPP and DPP were detected in nearly all urine samples ( $97 \%$ detect); however, geometric mean urine concentrations varied considerably from individual to individual. The distribution of BDCPP and DPP concentrations in urine samples is shown in Table $1(n=39)$. Levels of BDCPP and DPP at each time point were weakly correlated $\left(\mathrm{r}_{\mathrm{s}}=0.3, \mathrm{p}=0.1\right)$.

The rank order of women's urine concentrations was similar over time. Figure 1 shows the concentrations of BDCPP and DPP measured during the 18th week (average) and 28th week of pregnancy and shortly after their child's birth (one woman was missing a sample after the birth). Examining temporal variability in BDCPP and DPP during the 18th week of pregnancy using ICCs, we observed moderate to strong reliability (Table 2)(Rosner 2000). ICCs calculated over the entire course of pregnancy were slightly lower than those for samples taken over one week but results also suggest moderate reliability over time for urinary metabolites. In general, correcting for urine dilution using specific gravity decreased reliability estimates slightly.

Results of the analyses investigating the ability of a single sample to correctly identify a woman as highly exposed (based on her 3 month geometric mean metabolite level) are shown in Table 3. Sensitivity, specificity, positive predictive value, and negative predictive 
value were high for both metabolites (range 0.8 to 0.9 ), suggesting good agreement between methods of classifying women into the high exposure group (i.e. geometric mean throughout pregnancy or a single measurement). Results were similar when we used uncorrected concentrations (data not shown) and specific gravity corrected observations (Table 3).

\subsection{Discussion}

BDCPP and DPP were detected in nearly all urine samples collected from pregnant women in North Carolina, suggesting that exposures to TDCPP and TPP are widespread. Our results are consistent with several small studies which also reported variable, but near ubiquitous exposure (Cooper, Covaci et al. 2011, Carignan, McClean et al. 2013, Meeker, Cooper et al. 2013). However, geometric mean concentrations were notably higher in our small sample of pregnant women than those reported in any of the previous studies (approximately 3 to 10 times greater than concentrations reported by Carignan, McClean et al. (2013) or Meeker, Cooper et al. (2013)). Differences in excretion rates and kidney function during pregnancy may explain the higher metabolite levels observed in our study cohort relative to other nonpregnant cohorts.

We observed weak and non-statically significant correlations between urinary DPP and BDCPP concentrations which may be explained by differences in the sources of exposure to TDCPP and TPP. While both TDCPP and TPP are used as additive flame retardants in household products, TPP is also used as a plasticizer and lubricant and in hydraulic fluids (van der Veen and de Boer 2012). Differences in the metabolism and excretion of TDCPP and TPP also provide possible explanations for the weak correlation observed in urinary biomarkers of exposure.

Although the toxicokinetics of TDCPP and TPP in the human body have yet to be explored, data suggest that they are rapidly metabolized (to BDCPP and DPP, respectively) and are rapidly eliminated from the body (Lynn, Wong et al. 1981, Nomeir, Kato et al. 1981, Cooper, Covaci et al. 2011, Cooper, Getzinge et al. (Under Review)). Nonetheless, we observed moderate to strong reliability in the levels of BDCPP and DPP in urine samples collected throughout pregnancy and shortly after giving birth. Estimated ICCs for BDCPP and DPP were similar to those reported by Meeker et al. (2013) for urine samples collected from adult men over approximately 90 days. These ICCs were higher than those typically observed from rapidly metabolized compounds with primarily dietary sources (Braun, Kalkbrenner et al. 2011, Braun, Smith et al. 2012, Bradman, Kogut et al. 2013). For example, Braun et al. (2011) recently reported an ICC of 0.1 for bisphenol A in a cohort of pregnant women ( 3 samples from each woman collected at 16 and 26 weeks gestation and birth). These data suggest that TDCPP and TPP may come from more continuous sources of exposure than diet, such as contaminated dust in the home or workplace environments. This would seem a reasonable hypothesis given that human exposure to PBDE flame retardants in the US has been demonstrated to occur primarily from exposure to household dust, both in adults and in children (Stapleton, Eagle et al. 2012, Watkins, McClean et al. 2012).

Additionally, our results suggest that a single urine sample may reasonably capture the rank order of metabolite concentrations throughout pregnancy; the sensitivity and specificity for a single urine sample to predict the overall geometric mean were high for both BDCPP and DPP.

Adjustments for urine dilution have been recommended in the assessment of xenobiotics and are commonly included in epidemiologic investigations (Boeniger, Lowry et al. 1993). In this study, we chose to adjust with urinary specific gravity, which is thought to be less impacted by changes with age, body composition, physical activity, urine flow, time of day, diet, disease and pregnancy than other measures such as creatinine (Boeniger, Lowry et al. 
1993, Braun, Kalkbrenner et al. 2011). However, our results indicate that adjustment for SG generally decreased reliability over time, a finding that is consistent with previous assessments (Meeker, Cooper et al. 2013). There are several possible explanations for this pattern, including imperfect measures of urine dilution or insufficient methods of adjustment for dilution (e.g. Sorahan, Pang et al. 2008). Other measures of urine dilution and methods of correction should be included in future studies of BDCPP and DPP.

Inferences from this study are limited by the small sample size ( 8 women with a total of 39 measurements) and composition, specifically highly-educated pregnant women, which may differ substantially from other populations. However, our results are consistent with those from the only other study to assess the reliability of biomarkers in humans, in which the study population was composed of adult men (Meeker, Cooper et al. 2013). Follow-up of our findings in larger cohorts with longitudinal data will provide additional insights.

\title{
1.5 Conclusions
}

Our results demonstrate that exposure to TDCPP and TPP in pregnant women is likely. Additionally, we observed a high degree of variability in urinary metabolites of TDCPP and TPP (i.e. BDCPP and DPP) among women in our study population, but moderate to strong reliability of DPP and BDCPP in ranking women's exposure levels over time. Thus, while our analyses were limited by our small sample size and relatively homogeneous study population of North Carolina pregnant women, these data, provide some confidence that a single measure of levels during pregnancy may be sufficient in characterizing levels throughout pregnancy. Further data are needed to identify sources of exposure to OPFRs and to assess their impact on health.

\section{Acknowledgments}

This research was funded by the North Carolina Translational \& Clinical Science Institute Pilot Grant Program.

\author{
Abbreviations \\ BDCPP bis (1,3-dichloropropyl) phosphate \\ CI confidence interval \\ DPP diphenyl phosphate \\ GM geometric mean \\ ICC interclass correlation coefficient \\ IQR interquartile range \\ MDL method detection limit \\ ND non-detect \\ OPFRs organophosphate flame retardants \\ PBDEs polybrominated diphenyl ethers \\ SG specific gravity \\ TDCPP tris (1,3-dichloropropyl) phosphate \\ TPP triphenyl phosphate
}




\section{References}

Babich, MA. Preliminary Risk Assessment of Flame Retardant (FR) Chemicals in Upholstered Furniture Foam. Bethesda, MD 20814: U.S. Consumer Product Safety Commission; 2006.

Boeniger MF, Lowry LK, Rosenberg J. Interpretation of urine results used to assess chemical exposure with emphasis on creatinine adjustments: a review. Am Ind Hyg Assoc J. 1993; 54(10):615-627. [PubMed: 8237794]

Bradman A, Kogut K, Eisen EA, Jewell NP, Quiros-Alcala L, Castorina R, Chevrier J, Holland NT, Barr DB, Kavanagh-Baird G, Eskenazi B. Variability of organophosphorous pesticide metabolite levels in spot and 24-hr urine samples collected from young children during 1 week. Environ Health Perspect. 2013; 121(1):118-124. [PubMed: 23052012]

Braun JM, Kalkbrenner AE, Calafat AM, Bernert JT, Ye X, Silva MJ, Barr DB, Sathyanarayana S, Lanphear BP. Variability and predictors of urinary bisphenol A concentrations during pregnancy. Environ Health Perspect. 2011; 119(1):131-137. [PubMed: 21205581]

Braun JM, Smith KW, Williams PL, Calafat AM, Berry K, Ehrlich S, Hauser R. Variability of urinary phthalate metabolite and bisphenol A concentrations before and during pregnancy. Environ Health Perspect. 2012; 120(5):739-745. [PubMed: 22262702]

Carignan CC, McClean MD, Cooper EM, Watkins DJ, Fraser AJ, Heiger-Bernays W, Stapleton HM, Webster TF. Predictors of tris (1,3-dichloro-2-propyl) phosphate metabolite in the urine of office workers. Environ Int. 2013; 55:56-61. [PubMed: 23523854]

Cooper EM, Covaci A, van Nuijs AL, Webster TF, Stapleton HM. Analysis of the flame retardant metabolites bis(1,3-dichloro-2-propyl) phosphate (BDCPP) and diphenyl phosphate (DPP) in urine using liquid chromatography-tandem mass spectrometry. Anal Bioanal Chem. 2011; 401(7):21232132. [PubMed: 21830137]

Cooper, EM.; Getzinge, G.; Gooden, D.; Stapleton, HM. In vitro metabolism of the flame retardants tris (1,3-dichloro-2-propyl) phosphate (TDCPP) and triphenyl phosphate (TPP) by human liver. (Under Review)

Dishaw LV, Powers CM, Ryde IT, Roberts SC, Seidler FJ, Slotkin TA, Stapleton HM. Is the PentaBDE replacement, tris (1,3-dichloro-2-propyl) phosphate (TDCPP), a developmental neurotoxicant? Studies in PC12 cells. Toxicol Appl Pharmacol. 2011; 256(3):281-289. [PubMed: 21255595]

Hamer, R. [Accessed: August 14, 2013] INTRACC.SAS. Macro to calculate reliabilities for intraclass correlations. 1995. Available: http://support.sas.com/documentation/onlinedoc/stat/ex_code/121/ intracc.html

Hornung RW, Reed LD. Estimation of average concentration in the presence of nondetectable values. Appl Occup Environ Hyg. 1990; 5:46-51.

Lynn RK, Wong K, Garvie-Gould C, Kennish JM. Disposition of the flame retardant, tris(1,3dichloro-2-propyl) phosphate, in the rat. Drug Metab Dispos. 1981; 9(5):434-441. [PubMed: 6117442]

Mahalingaiah S, Meeker JD, Pearson KR, Calafat AM, Ye X, Petrozza J, Hauser R. Temporal variability and predictors of urinary bisphenol A concentrations in men and women. Environ Health Perspect. 2008; 116(2):173-178. [PubMed: 18288314]

Meeker JD, Cooper EM, Stapleton HM, Hauser R. Urinary metabolites of organophosphate flame retardants: temporal variability and correlations with house dust concentrations. Environ Health Perspect. 2013; 121(5):580-585. [PubMed: 23461877]

Meeker JD, Stapleton HM. House dust concentrations of organophosphate flame retardants in relation to hormone levels and semen quality parameters. Environ Health Perspect. 2010; 118(3):318-323. [PubMed: 20194068]

National Research Council (NRC). Toxicological Risks of Selected Flame-Retardant Chemicals. Subcommittee on Flame Retardant Chemicals; Washington, DC: National Research Council, National Academy of Sciences, National Academy Press; 2000.

Nomeir AA, Kato S, Matthews HB. The metabolism and disposition of tris(1,3-dichloro-2-propyl) phosphate (Fyrol FR-2) in the rat. Toxicol Appl Pharmacol. 1981; 57(3):401-413. [PubMed: 7222047] 
Rosner, B. Fundamentals of Biostatistics. 5. Pacific Grove, CA: Duxbury Press; 2000.

Shrout P, Fleiss J. Intra-class correlationsuses in assessing rater reliability. Psychological Bulletin. 1979; 86:420-428. [PubMed: 18839484]

Sorahan T, Pang D, Esmen N, Sadhra S. Urinary concentrations of toxic substances: an assessment of alternative approaches to adjusting for specific gravity. J Occup Environ Hyg. 2008; 5(11):721723. [PubMed: 18777412]

Stapleton HM, Allen JG, Kelly SM, Konstantinov A, Klosterhaus S, Watkins D, McClean MD, Webster TF. Alternate and new brominated flame retardants detected in U.S. house dust. Environ Sci Technol. 2008; 42(18):6910-6916. [PubMed: 18853808]

Stapleton HM, Eagle S, Sjodin A, Webster TF. Serum PBDEs in a North Carolina toddler cohort: associations with handwipes, house dust, and socioeconomic variables. Environ Health Perspect. 2012; 120(7):1049-1054. [PubMed: 22763040]

Stapleton HM, Klosterhaus S, Eagle S, Fuh J, Meeker JD, Blum A, Webster TF. Detection of organophosphate flame retardants in furniture foam and U.S. house dust. Environ Sci Technol. 2009; 43(19):7490-7495. [PubMed: 19848166]

Stapleton HM, Klosterhaus S, Keller A, Ferguson PL, van Bergen S, Cooper E, Webster TF, Blum A. Identification of flame retardants in polyurethane foam collected from baby products. Environ Sci Technol. 2011; 45(12):5323-5331. [PubMed: 21591615]

Stapleton HM, Sharma S, Getzinger G, Ferguson PL, Gabriel M, Webster TF, Blum A. Novel and high volume use flame retardants in US couches reflective of the 2005 PentaBDE phase out. Environ Sci Technol. 2012; 46(24):13432-13439. [PubMed: 23186002]

US Environmental Protection Agency (EPA). Furniture Flame Retardancy Partnership: Environmental Profiles of Chemical Flame-Retardant Alternative for Low-Density Polyurethane Foam. Washington, DC: 2005. EPA 742-R-05-002

van der Veen I, de Boer J. Phosphorus flame retardants: properties, production, environmental occurrence, toxicity and analysis. Chemosphere. 2012; 88(10):1119-1153. [PubMed: 22537891]

Watkins DJ, McClean MD, Fraser AJ, Weinberg J, Stapleton HM, Sjodin A, Webster TF. Impact of dust from multiple microenvironments and diet on PentaBDE body burden. Environ Sci Technol. 2012; 46(2):1192-1200. [PubMed: 22142368] 


\section{Highlights}

Exposures to TDCPP and TPP are widespread and variable for pregnant women. TDCPP and TPP metabolites were detected frequently in urine samples.

Measures of TDCPP and TPP metabolites were moderately reliable throughout pregnancy.

A single measure of metabolites captured information on the rank order of exposure over time. 

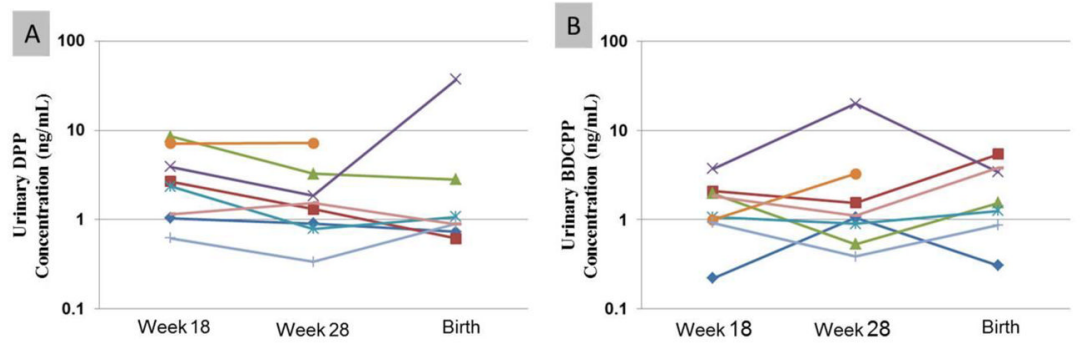

Figure 1.

Concentrations $(\mathrm{ng} / \mathrm{mL})$ of metabolites in urine from repeated samples collected from 8 pregnant women in the18th (averaged across three measurements) and 28th weeks of pregnancy and at the time of birth. (A) DPP; (B) BDCPP. Colors represent individual participants. Measured samples are connected with lines to assist in the interpretation of changes over time. 


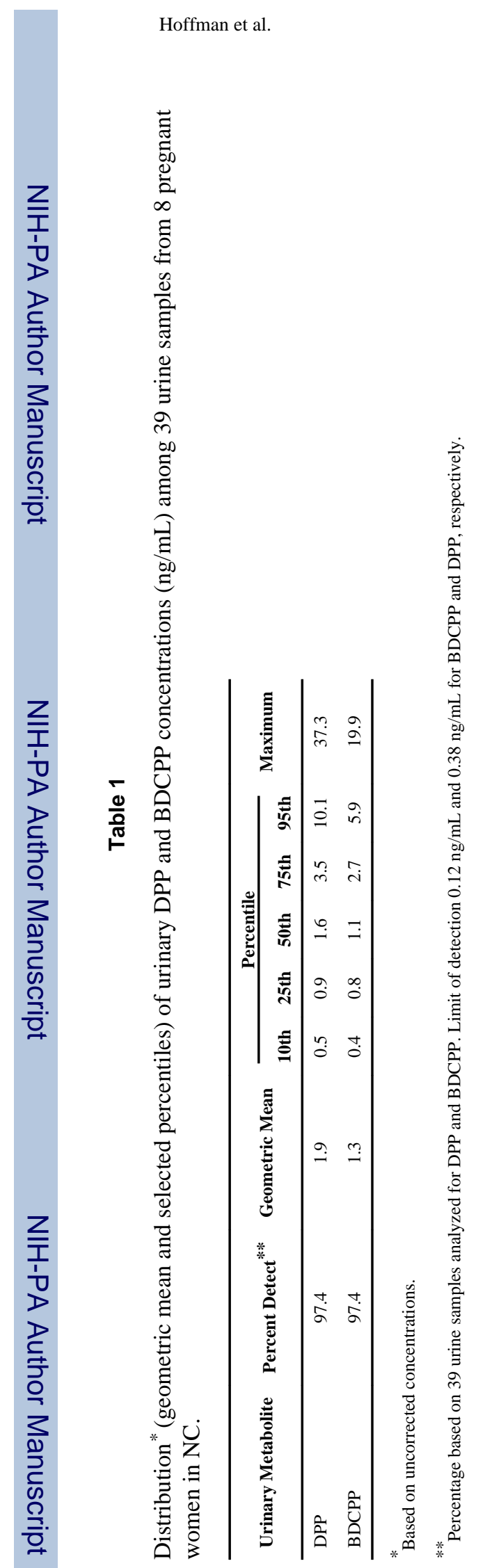

Environ Int. Author manuscript; available in PMC 2015 February 01. 


\section{Table 2}

Interclass correlation coefficients (ICC) for uncorrected and specific gravity-corrected urinary BDCPP and DPP concentrations for 39 samples among 8 pregnant $\mathrm{NC}$ women.

\begin{tabular}{lcccc}
\hline \multirow{2}{*}{ Urinary Metabolite } & \multicolumn{2}{c}{ Week 18 Samples $(\mathbf{n = 2 4})$} & \multicolumn{2}{c}{ All Samples $(\mathbf{n}=\mathbf{2 3})$} \\
& Uncorrected & SG $^{*}$-corrected & Uncorrected & SG $^{*}$-corrected \\
\hline DPP & $0.7(0.5,0.8)$ & $0.6(0.4,0.7)$ & $0.6(0.4,0.7)$ & $0.4(0.2,0.6)$ \\
BDCPP & $0.5(0.4,0.7)$ & $0.6(0.4,0.7)$ & $0.5(0.4,0.7)$ & $0.4(0.2,0.6)$ \\
\hline * SG-Specific gravity & & & & \\
\end{tabular}




\section{Table 3}

Sensitivity and specificity analysis for the ability of a urine measurement at 18 week, 28 weeks or after birth, to correctly classify a high exposure group according $\mathrm{GM}^{*}$ metabolite concentration throughout pregnancy among $8 \mathrm{NC}$ women.

\begin{tabular}{lcccc}
\hline Urinary Metabolite & Sensitivity & Specificity & Positive Predictive Value & Negative Predictive Value \\
\hline DPP & 0.8 & 0.9 & 0.9 & 0.8 \\
BDCPP & 0.8 & 0.8 & 0.8 & 0.8 \\
\hline * GM-Geometric Mean & & & &
\end{tabular}

